\title{
Coagulase-negative staphylococci in outpatient routines: the implications of switching from CLSI to BrCAST/EUCAST guidelines
}

\author{
Vinícius Pietta Perez ${ }^{1}$. Jéssica Karoliny Baptista Porto Carvalho ${ }^{2} \cdot$ Marianne Schrader de Oliveira $^{3}$. \\ Adriana Medianeira Rossato ${ }^{4}$. Caroline Dani ${ }^{2} \cdot$ Gertrudes Corção ${ }^{5}$. Pedro Alves d'Azevedo ${ }^{4}$
}

Received: 5 September 2019 / Accepted: 16 April 2020 / Published online: 23 April 2020

(C) Sociedade Brasileira de Microbiologia 2020

\begin{abstract}
Coagulase-negative staphylococci (CoNS) are frequently isolated in clinical specimens and are important reservoirs of resistance genes. In 2019, the Brazilian government set the BrCAST/EUCAST (Brazilian Committee on Antimicrobial Susceptibility Testing) guidelines as the national standard, resulting in changes in the interpretation of CoNS susceptibility tests. From outpatients, disk-diffusion susceptibility of 65 CoNS cultures were evaluated and compared using classification criteria from both CLSI and BrCAST/EUCAST. The isolates were identified using matrix assisted laser desorption ionization-time of flight (MALDI-TOF), and the presence of the mecA gene was determined. The most prevalent species were Staphylococcus saprophyticus (32.3\%), S. haemolyticus (18.5\%), and S. epidermidis $(9.2 \%)$. Almost perfect agreement was seen between the guidelines, except concerning oxacillin and gentamicin, and the prevalence of multidrug resistant isolates increased with the use of BrCAST/EUCAST. Of all, 15 (23.1\%) isolates, mainly S. epidermidis and S. haemolyticus, were positive for the mecA gene, and only three were detected when using CLSI or BrCAST/EUCAST disk-diffusion screening. This, using either guideline, could reveal the difficulty of determining oxacillin resistance. Using warning zones or molecular methods might well be indicated for CoNS. In conclusion, adoption of the BrCAST/EUCAST guidelines will result in certain artificial changes in epidemiological susceptibility profiles, and clinicians and institutions should be aware of the possible implications.
\end{abstract}

Keywords Antimicrobial susceptibility testing · Brazilian Committee on Antimicrobial Susceptibility Testing · European Committee on Antimicrobial Susceptibility Testing · Clinical Laboratory Standards Institute $\cdot$ Coagulase-negative staphylococci $\cdot$ Oxacillin resistance

Responsible Editor: Afonso Luis Barth.

Electronic supplementary material The online version of this article (https://doi.org/10.1007/s42770-020-00278-1) contains supplementary material, which is available to authorized users.

Vinícius Pietta Perez

vinicius@ccs.ufpb.br

1 Departamento de Fisiologia e Patologia, Universidade Federal da Paraíba, Campus I - Cidade Universitaria, João Pessoa, PB 58051-900, Brazil

2 Curso de Biomedicina, Centro Universitário Metodista IPA, Porto Alegre, Brazil

3 Laboratório Endocrimeta, Porto Alegre, Brazil

4 Programa de Pós-Graduação em Ciências da Saúde, Universidade Federal de Ciências da Saúde de Porto Alegre, Porto Alegre, Brazil

5 Departamento de Microbiologia, Imunologia e Parasitologia, Universidade Federal do Rio Grande do Sul, Porto Alegre, Brazil

\section{Introduction}

Coagulase-negative staphylococci (CoNS), mainly in skin and mucous membranes, are a normal part of the human microbiota. However, for many years, the bacteria had been underrated as infectious agents, nowadays the pathogenic potential of CoNS is well established, and they have been associated with important opportunistic infections and multiple antibiotic resistance [1]. CoNS are a heterogeneous group consisting of at least 39 species divided phylogenetically into 14 cluster groups, where identification by classical phenotypic tests is difficult [2]. Although certain species remain susceptible to various antibiotics, some strains are frequently associated with resistance to multiple antistaphylococcal agents. The increase of oxacillin resistant CoNS - as mediated by penicillin-binding protein (PBP2a) modifications, encoded by the mecA gene- has become a pressing concern $[1,3]$. 
The Clinical and Laboratory Standards Institute (CLSI) M100 document (1986), originally from the former National Committee for Clinical Laboratory Standards (NCCLS), is worldwide one of the most used guidelines for interpretation of antimicrobial susceptibility testing (AST) [4]. In 2005, the Brazilian National Health Surveillance Agency (ANVISA) obtained permission from CLSI to distribute a translated version of M100-S15 to clinical laboratories [5], and henceforth CLSI - M100-S15 became the principal guide used in Brazil. Some laboratories, due to language barriers or lack of resources to purchase annually updated versions, still use older, outdated versions of M100-S15. In resource-poor settings (like Brazilian laboratories), there is a need for suitable and free AST guidelines. In 2019, after intense discussions by a group of experts contracted by the Brazilian Ministry of Health, the Brazilian Committee on Antimicrobial Susceptibility Testing (BrCAST) guidelines were set as the new standard for AST in Brazil [6].

In 2013, various Brazilian scientific societies united to form BrCAST, aiming at consensus AST breakpoints for Brazil. This is because, at the time, there were no CLSI breakpoints for colistin or tigecycline and there were also reported emergences of KPC-2 producing $K$. pneumoniae. The BrCAST guidelines are based on the European Committee on Antimicrobial Susceptibility Testing (EUCAST) breakpoints, translated to Portuguese, and freely available on their website [7]. EUCAST bases its clinical breakpoints on epidemiological cut-off values and pharmacokinetic-pharmacodynamic properties. Unlike CLSI, the General Committee of EUCAST has representatives in several countries including Brazil, and the pharmaceutical industry retains only a consultative role [8].

The aim of this study was to determine the CoNS species frequency in samples of an outpatient population using both the CLSI and the BrCAST/EUCAST AST guidelines and to then compare the susceptibility/resistance rates of selected antibiotics.

\section{Materials and methods}

The study included strains previously identified as CoNS from outpatients of a clinical laboratory in Porto Alegre, Southern Brazil, during the period of from March to August 2018. For fast bacterial identification, isolates were plated on MuellerHinton agar, and matrix-assisted laser desorption ionizationtime of flight (MALDI-TOF) methodology was performed out in the Microflex LT (Bruker Corporation, USA) platform according to the manufacturer's specifications for direct colony identification. The spectra were analyzed using MALDI Biotyper 4.0 software and standard pattern matching with a default setting in accordance with previously studies [9].
For susceptibility testing, the disk diffusion method according to Kirby-Bauer was used [10]. Antimicrobial susceptibility for oxacillin using a cefoxitin $30 \mu \mathrm{g}$ disk (CFO), ciprofloxacin $5 \mu \mathrm{g}(\mathrm{CIP})$, norfloxacin $10 \mu \mathrm{g}$ (NOR), gentamicin $10 \mu \mathrm{g}$ (GEN), erythromycin $15 \mu \mathrm{g}$ (ERY), clindamycin $2 \mu \mathrm{g}$ (CLI), trimethoprim-sulfamethoxazole 1.25/23.75 $\mu \mathrm{g}$ (TMP/SMX), and chloramphenicol $30 \mu \mathrm{g}$ (CLO) (SENSIDISC, DME, Brazil) was performed on Mueller-Hinton agar (TM Media, Titan Biotech, India) and using a suspension equivalent to MacFarland 0.5 from overnight cultures followed by incubation at $35 \pm 1{ }^{\circ} \mathrm{C}$ for 20 and $24 \mathrm{~h}$. Inhibition zone diameters were determined and interpreted according to the CLSI 2019, BrCAST/EUCAST 2019, and CLSI 2018 guidelines for norfloxacin (see supplementary file 1) [4, 11, 12].

Multiplexed PCR for detection of the mecA gene and determination of $\mathrm{SCCmec}$ type was performed for the isolates as previously described [13].

For statistical analysis, the percentage concordance rate between the two guidelines was compared. Cohen's Kappa coefficient was used to measure the guideline agreement rate for each antimicrobial; $p \leq 0.05$ was considered statistically significant, and all analyses were done using IBM SPSS version 20.0 for macOS (IBM Corporation, USA). Also, the research protocol was approved by an ethics committee.

\section{Results}

A total of 65 CoNS strains were included in this study, $90.7 \%$ of the isolates were obtained from urine, $6.2 \%$ from blood, and $3.1 \%$ from secretions. The average patient age was $41 \pm$ 23.2 years (ranging from 9 to 89 ), and the majority of patients $52 / 63(80 \%)$ were female.

Within the overall sample population, the MALDI-TOF methodology for rapid identification revealed the presence of at least seven CoNS species. Of all, 47 (72.3\%) of the isolates were consistently identified at the species level. The remainder 18 (27.7\%) did not fulfill the conditions for identification at the species level, being thus identified as Staphylococcus spp., of these, eight were best matched with $S$. saprophyticus, four with $S$. haemolyticus, three with S. lugdunensis, two with $S$. epidermidis, and one with S. cohnii. Table 1 summarizes the CoNS species identified according to sample origin.

In the antimicrobial susceptibility testing (AST), the specie-antibiotic susceptibilities were equal in both guidelines, agreement for each antibiotic ranged from 89.1 to $100 \%$, with noted susceptibilities in both guidelines of 96.9\% for CLO, $86.2 \%$ for CIP, $81.5 \%$ for CLI, $49.2 \%$ for ERY, $86.8 \%$ for NOR, and $63.1 \%$ for TMP/SMX. On the other hand, for GEN we observed a susceptibility of $93.8 \%$ with CLSI and $84.4 \%$ with BrCAST/EUCAST. For oxacillin, we observed a susceptibility of $93.8 \%$ with CLSI and of 
Table 1 Species of CoNS species identified according to sample origin

\begin{tabular}{lcllc}
\hline Identification & Urine & Blood & Secretion & Total (\%) \\
\hline S. saprophyticus & 21 & 0 & 0 & $21(32.3)$ \\
S. haemolyticus & 12 & 0 & 0 & $12(18.5)$ \\
S. epidermidis & 2 & 4 & 0 & $6(9.2)$ \\
S. warneri & 3 & 0 & 1 & $4(6.2)$ \\
S. lugdunensis & 2 & 0 & 0 & $2(3.1)$ \\
S. caprae & 1 & 0 & 0 & $1(1.5)$ \\
S. condimenti & 1 & 0 & 0 & $1(1.5)$ \\
Staphylococcus spp. & 17 & 0 & 1 & $18(27.7)$ \\
\hline
\end{tabular}

96.9\% using the BrCAST/EUCAST criteria. Kappa analysis presented perfect agreement for CLO and CLI $(K=1)$, and almost perfect agreement for CIP $(K=0.936)$, ERY $(K=$ $0.972)$, NOR $(K=0.887)$, and TMP/SMX $(K=0.968)$. Substantial agreement was observed with oxacillin using a CFO disk $(K=0.652)$, and only moderate agreement was observed for GEN ( $K=0.458$ ). Comparing the test results using both guidelines, we noted five minor divergences; in which CLSI classified isolates as intermediate and BrCAST/ EUCAST classified isolates as resistant. Six major divergences were observed for GEN, being classified as susceptible by CLSI and yet resistant by BrCAST/EUCAST. For the oxacillin criteria, the results of two isolates; $S$. haemolyticus and S. saprophyticus were considered to be major errors, leading to classification by CLSI as resistant, and by BrCAST/ EUCAST as susceptible. Table 2 summarizes the susceptibilities, agreement, and kappa statistics for both guidelines. Of the 65 CoNS tested, 10 (15.4\%) - CLSI and 14 (21.9\%) BrCAST/EUCAST were resistant to at least three antimicrobial classes and were therefore classified as multidrug resistant (MDR) [9] with a concordance of $93.8 \%$ (Table 3).
Positives for the mecA gene were observed in 15 isolates (23\%) (Table 4), three were classified as resistant by at least one guideline, and $12(18.5 \%)$ were classified as susceptible, an error for both guidelines. Figure 1 presents the distributions of the CFO disk inhibition zone diameters observed in the study by criteria for mecA screening of staphylococci using CLSI and BrCAST/EUCAST. Inducible resistance to CLI was observed as positive for seven isolates, (two $S$. saprophyticus, two $S$. warneri, one $S$. epidermidis, one $S$. haemolyticus, and one Staphylococcus sp.), when using D test - (disk approximation test) detection.

\section{Discussion}

Due to the high association of CoNS with healthcare-acquired (nosocomial) infections [14, 15], there are few reports concerning epidemiology in outpatients. Our study did not include patients from healthcare facilities, and the samples were composed mainly of urine specimens. Indeed, some CoNS are frequent uropathogens, such as $S$. saprophyticus in young and middle-aged women [16]. Before MALDI-TOF became available, identification of CoNS at the species level was not routinely performed [14]. In urine specimens of a like population of patients in Ghana, a high prevalence of S. haemolyticus (75\%), followed by S. epidermidis (13\%) and S. hominis, and as in our study S. warneri, S. lugdunensis, and S. condimenti [17], was also observed, yet surprisingly $S$. saprophyticus was not reported. This might be indicative of a greater diversity of CoNS species in outpatients and of a possible rise of unfamiliar isolates such as $S$. condimenti, which was first reported as a pathogen in 2014 [18]. The data suggest that the S. epidermidis-like group, specially $S$. haemolyticus, seems to have an important role as gram-positive uropathogens in both patients and outpatients $[14$, $17,19]$.

Table 2 Susceptibilities of CoNS to antibiotics, with concordance and kappa statistics for the CLSI and BrCAST/EUCAST guidelines

\begin{tabular}{|c|c|c|c|c|c|c|c|c|c|}
\hline \multirow[t]{2}{*}{ Antimicrobial agent } & \multicolumn{3}{|l|}{ CLSI } & \multicolumn{3}{|c|}{ BrCAST/EUCAST } & \multirow[t]{2}{*}{ Concordance $\%$} & \multirow[t]{2}{*}{ Kappa } & \multirow[t]{2}{*}{$P$} \\
\hline & $\mathrm{S}(\%)$ & $\mathrm{I}(\%)$ & $\mathrm{R}(\%)$ & $\mathrm{S}(\%)$ & $\mathrm{I}(\%)$ & $\mathrm{R}(\%)$ & & & \\
\hline Chloramphenicol & $62(96.9)$ & 0 & $2(3.1)$ & $62(96.9)$ & 0 & $2(3.1)$ & $100.0 \%$ & 1 & $<0.000$ \\
\hline Ciprofloxacin & $56(86.2)$ & $1(1.5)$ & $8(12.3)$ & $56(86.2)$ & 0 & $9(13.8)$ & $98.5 \%$ & $0.936( \pm 0.060)$ & $<0.000$ \\
\hline Clindamycin & $53(81.5)$ & 0 & $12(18.5)$ & $53(81.5)$ & 0 & $12(18.5)$ & $100.0 \%$ & 1 & $<0.000$ \\
\hline Erythromycin & $32(49.2)$ & $4(6.2)$ & $29(44.6)$ & $32(49.2)$ & $3(4.6)$ & $30(46.2)$ & $98.5 \%$ & $0.972( \pm 0.028)$ & $<0.000$ \\
\hline Gentamicin & $60(93.8)$ & $1(1.6)$ & $3(4.7)$ & $54(84.4)$ & 0 & $10(15.6)$ & $89.1 \%$ & $0.458( \pm 0.151)$ & $<0.000$ \\
\hline Norfloxacin & $33(86.8)$ & $1(2.6)$ & $4(10.5)$ & $33(86.8)$ & 0 & $5(13.2)$ & $97.4 \%$ & $0.887( \pm 0.100)$ & $<0.000$ \\
\hline Oxacillin* & $61(93.8)$ & 0 & $4(6.2)$ & $63(96.9)$ & 0 & $2(3.1)$ & $96.9 \%$ & $0.652( \pm 0.227)$ & $<0.000$ \\
\hline Trimethoprim-sulfamethoxazole & $41(63.1)$ & $2(3.1)$ & $22(33.8)$ & $41(63.1)$ & $1(1.5)$ & $23(35.4)$ & $98.5 \%$ & $0.968( \pm 0.031)$ & $<0.000$ \\
\hline
\end{tabular}

$I$ intermediate, $R$ resistant, $S$ sensible

*Cefoxitin disk 
Table 3 Susceptibilities profiles of MDR CoNS according to CLSI and BrCAST/EUCAST guidelines

\begin{tabular}{|c|c|c|c|c|}
\hline \multirow[t]{2}{*}{ Species } & \multicolumn{2}{|l|}{ CLSI } & \multicolumn{2}{|l|}{ BrCAST/EUCAST } \\
\hline & Number of isolates & Antimicrobial resistance profiles & Number of isolates & Antimicrobial resistance profiles \\
\hline S. epidermidis & 3 & $\begin{array}{l}\text { OXA - CIP - TMP/SMX } \\
\text { CLI - ERY - CIP } \\
\text { CLI - ERI - CIP - TMP/SMX }\end{array}$ & 4 & $\begin{array}{l}\text { OXA - CIP - GEN - TMP/SMX } \\
\text { CLI - ERY - CIP } \\
\text { CLI - ERI - CIP - TMP/SMX } \\
\text { GEN - TMP/SMX - NOR }\end{array}$ \\
\hline S. haemolyticus & 2 & $\begin{array}{l}\text { CLI - ERY - GEN - NOR } \\
\text { ERY - GEN - TMP/SMX }\end{array}$ & 5 & $\begin{array}{l}\text { CLI - ERY - GEN - NOR } \\
\text { ERY - GEN - TMP/SMX } \\
\text { ERY - CIP - GEN - TMP/SMX } \\
\text { OXA - ERY - GEN } \\
\text { CIP - GEN - TMP/SMX - NOR }\end{array}$ \\
\hline S. warneri & 2 & $\begin{array}{l}\text { CLI - ERY - TMP/SMX } \\
\text { CLI - ERY - TMP/SMX }\end{array}$ & 2 & $\begin{array}{l}\text { CLI - ERY - TMP/SMX } \\
\text { CLI - ERY - TMP/SMX }\end{array}$ \\
\hline S. saprophyticus & 1 & OXA - CLI - ERY - TMP/SMX & 1 & CLI - ERY - TMP/SMX \\
\hline Staphylococcus spp. & 2 & $\begin{array}{l}\text { ERY - CIP - TMP/SMX -NOR } \\
\text { CLI - ERY - TMP/SMX }\end{array}$ & 2 & $\begin{array}{l}\text { ERY - CIP - GEN- TMP/SMX -NOR } \\
\text { CLI - ERY - TMP/SMX }\end{array}$ \\
\hline
\end{tabular}

$C L I$ clindamycin, $C I P$ ciprofloxacin, $E R Y$ erythromycin, GEN gentamicin, NOR norfloxacin, $O X A$ oxacillin, $T M P / S M X$ trimethoprim-sulfamethoxazole

Though CoNS present fewer virulence factors than $S$. aureus, they still maintain an active role in antimicrobial resistance, serving as reservoirs of many resistance genes and contributing to the spreading of multiresistance between staphylococci [20]. That being said, the determination of an accurate CoNS susceptibility profile should carefully pursued. CLSI, despite its cost and remaining untranslated to Portuguese, has been the standard guideline in Brazilian laboratories for interpreting AST. With AST, it is critical for guidelines to be up to date, free, and readily available. Since 2019, the Brazilian Ministry of Health has adopted BrCAST/ EUCAST as the standard guideline for Brazilian laboratories [6], yet CLSI and BrCAST/EUCAST methods lead to differing disk diffusion breakpoints and consequently divergent epidemiological susceptibility profiles.

CLSI and EUCAST susceptibility profiles have been compared in studies, and significant changes have been reported concerning certain pathogens [21-26]. Due to its more restrictive breakpoints, lower susceptibility rates have been observed for EUCAST [21]. However, the impact of this change on CoNS susceptibility profiles has not been fully explored, and in our comparison study, most CoNS-antibiotic combinations presented perfect or almost perfect agreement (except for GEN and Oxacillin).

Several countries have been progressively moving to implement EUCAST as a standard AST guideline, and for $S$. aureus, (excluding use of aminoglycosides) some studies evaluating the impact of this change in staphylococci show excellent correlations between both guidelines. The use of EUCAST criteria for minimal inhibitory concentration (MIC) results in a significant reduction in GEN susceptibility in S. aureus [22-24]. One study with CoNS observed the same for $S$. haemolyticus but not for $S$. lugdunensis. A plausible explanation is the lower number of resistance mechanisms observed in S. lugdunensis [25]. The contrast observed for GEN is due to the more stringent BrCAST/EUCAST breakpoint, which leads to a higher resistance rate for staphylococci. The CLSI breakpoint for GEN for staphylococci is $\geq$
Table 4 Distribution of SCCmec types according to CoNS species

\begin{tabular}{lclll}
\hline Species & Number of mecA-positive isolates & \multicolumn{3}{l}{ SCCmec type } \\
\cline { 3 - 5 } & & III & IV & V \\
\hline S. epidermidis & 5 & 0 & 3 & 2 \\
S. haemolyticus & 5 & 1 & 3 & 1 \\
S. saprophyticus & 1 & 0 & 1 & 0 \\
Staphylococcus spp. & 4 & 1 & 1 & 2 \\
Total & 15 & $2(13.3 \%)$ & $8(53.3 \%)$ & $5(33.3 \%)$ \\
\hline
\end{tabular}




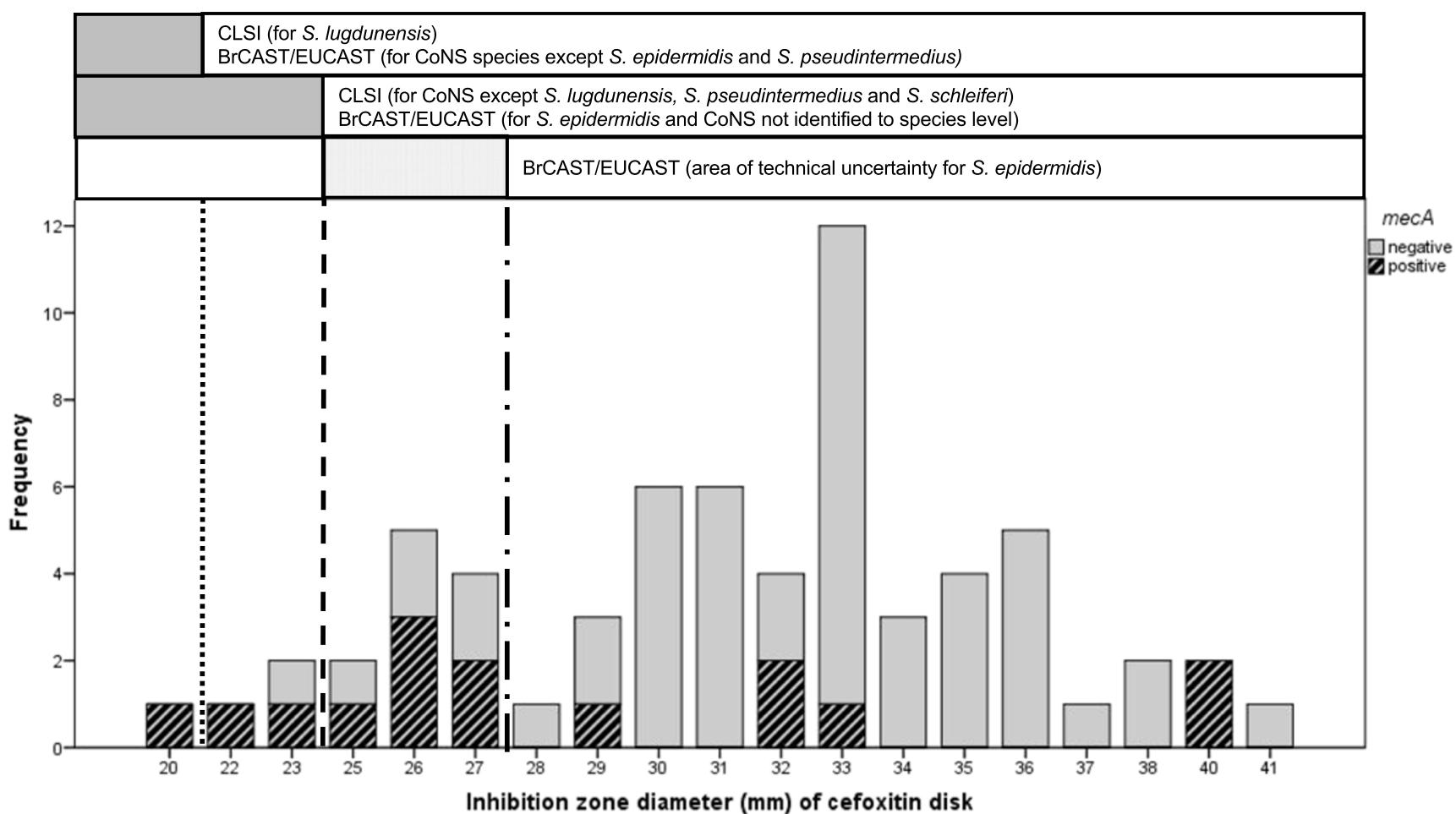

Fig. 1 Distribution of inhibition zone diameters for CoNS and screening criteria for mecA gene by CLSI and BrCAST/EUCAST

$15 \mathrm{~mm}$ [4], conversely, the BrCAST/EUCAST breakpoint for CoNS is $\geq 22 \mathrm{~mm}$. Currently, BrCAST/EUCAST has a less rigorous GEN breakpoint for S. aureus [12], and a review of CoNS breakpoints might be in order to prevent false, BrCAST/EUCAST-related epidemiological profile changes in Brazil.

Organizing disagreements into minor and major divergences, we found five minor divergences between the guidelines. In each case, CLSI classified the isolate as intermediate, whereas BrCAST/EUCAST classified the isolate as resistant. In three cases, this resulted in an MDR classification. Disagreement for ERY as an S. haemolyticus isolate resistant to oxacillin was observed, and a different $S$. haemolyticus presented disagreement for CIP. Discordances were also noted for GEN and NOR against another $S$. epidermidis. Reclassification to MDR can change regional epidemiological profiles and influence antimicrobial selection by clinicians.

Even though there is perfect agreement observed between the guidelines in the literature for oxacillin MICs in staphylococci [24-26], the disk diffusion method remains the standard tool for detecting oxacillin resistance and the changes between the guidelines are relevant. Oxacillin resistance is used as a predictor for the presence of the mecA gene and consequently of resistance to beta-lactams, (penicillins, most cephalosporins, and carbapenems), this may well affect patient management. CLSI requires differing conditions according to species, with incubation of $16-18 \mathrm{~h}$ and using a cefoxitin $30-\mu \mathrm{g}$ disk with a cut-off of $\leq 21 \mathrm{~mm}$ for $S$. lugdunensis; for $S$. epidermidis and other CoNS, it requires an incubation of
$24 \mathrm{~h}$ and cut-off of $\leq 24 \mathrm{~mm}$ (except S. pseudintermedius and S. schleiferi). An oxacillin 1- $\mu \mathrm{g}$ disk with cut-offs of $\leq 17 \mathrm{~mm}$ is mandatory for S. pseudintermedius and S. schleiferi yet can also be used for S. epidermidis [4]. On the other hand, for all CoNS species except $S$. epidermidis and S. pseudintermedius (e.g. S. capitis, S. cohnii, S. haemolyticus, S. hominis, S. hyicus, S. lugdunensis, S. saprophyticus, S. schleiferi, S. sciuri, S. simulans, S. warneri and S. xylosus), BrCAST/ EUCAST requires incubations of $18 \pm 2 \mathrm{~h}$, and a cefoxitin $30-\mu \mathrm{g}$ disk with a cut-off of $<22 \mathrm{~mm}$. For $S$. epidermidis and non-species level identified CoNS, BrCAST/EUCAST requires a cut-off of $<25 \mathrm{~mm}$. For $S$. pseudintermedius screening, BrCAST/EUCAST requires use of a $1-\mu \mathrm{g}$ oxacillin disk and a cut-off of $<20 \mathrm{~mm}$ [12].

In our results, four CoNS were classified as oxacillin resistant by CLSI and two by BrCAST/EUCAST, revealing only partial agreement. Despite the specific conditions for $S$. epidermidis in BrCAST/EUCAST, other species included in the $S$. epidermidis-like group, such as $S$. haemolyticus, are not covered in this criteria, and one $m e c A$-positive isolate with a borderline inhibition zone detected by CLSI was classified as oxacillin susceptible by BrCAST/EUCAST. In fact, $S$. haemolyticus is frequently MDR $[20,25]$ developing resistance to beta-lactams. In our sample, $41.7 \%$ harbored mecA, three of these (with inhibition zones of $26 \mathrm{~mm}, 26 \mathrm{~mm}$, and $29 \mathrm{~mm}$ ) were not detected using the disk diffusion screening criteria of either guideline. In a previous study with Brazilian isolates, approximately $60 \%$ of the community isolates and $100 \%$ of the blood isolates were positive for mecA [27]. 
CLSI and BrCAST/EUCAST have different breakpoints for oxacillin MIC and disk diffusion in S. saprophyticus (respectively $0.5 \mathrm{mg} / \mathrm{L}$ and $\leq 24 \mathrm{~mm}$; and $2.0 \mathrm{mg} / \mathrm{L}$ and $<$ $22 \mathrm{~mm}$,) [4, 12]. One mecA-negative isolate was classified as resistant by CLSI, and one mecA-positive isolate was classified as susceptible by both guidelines. For S. saprophyticus, oxacillin resistance needs to be careful evaluated since previous studies have observed differing results from the guideline criteria for MIC, disk diffusion, and mecA presence [27, 28].

The presence of the mecA gene in S. epidermidis was high $(83 \%)$, but only one of five isolates was detected using the screening criteria for disk diffusion. The isolates classified as oxacillin susceptible presented large CFO disk inhibition zones (27 to $40 \mathrm{~mm}$ ), and only one isolate met the suggested BrCAST/EUCAST confirmation criteria [12].

In resource-poor laboratory settings, CoNS are frequently not identified at the species level due to the limitations of classical phenotypic tests [14]. In our sample, even with the use of MALDI-TOF, $27.7 \%$ of isolates were not confidently identified at the species level, thus the importance of evaluating the CLSI and BrCAST/EUCAST guidelines for determination of oxacillin resistance for Staphylococcus spp. According to CLSI, using the CFO disk is an acceptable method for Staphylococcus spp. or oxacillin MIC. However, the MIC may emphasize resistance, and results between 0.5 and $2 \mathrm{mg} / \mathrm{L}$ must be tested for mecA [4]. BrCAST/EUCAST indicates using the $\mathrm{CFO}$ disk with no recommendations of MIC breakpoints for CoNS not identified at species level [12]. Of 18 CoNS not identified at the species level, four (22\%) - best matched to $S$. haemolyticus-harbored the mecA gene, and both guidelines failed in screening for this resistance mechanism.

It is also important to note that the oxacillin $1-\mu \mathrm{g}$ disk is recommended by CLSI for $S$. pseudintermedius and S. schleiferi and by BrCAST/EUCAST for $S$. pseudintermedius (cut-off $\leq 17 \mathrm{~mm}$ and $<20 \mathrm{~mm}$, respectively) to screen for mecA. CLSI also recommended oxacillin MIC as an acceptable methodology for all CoNS $[4,12]$. Despite this consideration, in our sample we did not identify these two species.

The vast majority of clinical CoNS possess the mobile genetic element $S C C m e c$ that harbors the mec gene [2]. Detection of oxacillin resistance in these isolates is a huge challenge since agreement between phenotypic and molecular methods oscillates among strains. Pinheiro et al. [27] attributes this to resistance heterogeneity, with borderline resistance being a very common phenomenon. In 2019, BrCAST/ EUCAST introduced a warning zone $(25-27 \mathrm{~mm})$ for $S$. epidermidis called the Area of Technical Uncertainty (ATU) indicating the need of confirming susceptibility categorization, by using an alternative test [12]. The adoption of the ATU warning for all CoNS might increase oxacillin resistance detections in our study, especially in $S$. haemolyticus isolates.
MDR strains frequently acquire resistance to macrolides and related antibiotics. Indeed, some SCCmec harbor genes for resistance to ERY and others antibiotics [2]. Macrolides are not used for first-line treatment of urinary infections, and the high rates of acquired ERY resistance observed could indicate a change in the human microbiota due to the overuse of these agents in the community. The mutation in $23 \mathrm{~S}$ rRNA mediated by the erm gene is the most frequently noted mechanism of resistance to ERY, and the presence of these genetic elements generally results in a phenotype of resistance to macrolides, lincosamides, and streptogramin B (MLS), which can be either constitutive (cMLS) or inducible (iMLS). As other mechanisms, efflux pumps encoded by the msrA gene mediate resistance to MLS, and drug modification is encoded by the lnu gene [29]. The iMLS phenotype occurs in strains that carry erm genes but are susceptible to CLI in AST. Clindamycin treatment in patients with iMLS resistance may lead to therapeutic failure [30], and the disk approximation test, or D test, is used to detect this phenotype. Of the isolates resistant to macrolides, 7/30 (23.3\%) were positive to phenotype iMLS. For the cMLS phenotype, 3/30 (10\%) were positive, with 20/ $30(66.6 \%)$ of the isolates being resistant only to macrolides. All isolates resistant to CLI were also resistant to ERY. The prevalence of macrolide-resistant phenotypes in CoNS might vary according to regions and populations that use macrolides; in fact, our prevalence for iMLS was greater than previous studies around the world, 11.8 to $15.2 \%$ [29-31].

There are few studies evaluating the use of CLSI and EUCAST guidelines for CoNS, and our study presents some limitations. First, the limited sample size in a single laboratory may interfere in the epidemiology. The AST comparison results are limited to antibiotics where both guidelines indicated the same disk concentrations. And also, we did not evaluate nitrofurantoin (important in treating urinary infections), penicillin, ampicillin, or linezolid. Our purpose was to evaluate the implications of switching AST guidelines and not to design an experiment establishing which presents better performance in determining susceptibility to oxacillin. However, evaluation of oxacillin disk performance, or the oxacillin MICs for all CoNS, could be important data for analysis of this issue. Finally, we did not use molecular typing tools such as MLST or PFGE to evaluate clonality.

CoNS are often underestimated as etiological agents of human infections, especially in outpatients. The introduction of MALDI-TOF led to recognition of the great species diversity; and that $S$. haemolyticus seems to have an important role as urinary pathogen with the ability to accumulate antibiotic resistant determinants. Our research revealed acceptable concordance between CLSI and BrCAST/EUCAST except when using aminoglycosides. We note that determination of oxacillin susceptibility in CoNS has many pitfalls for both guidelines. When investigating oxacillin resistance, the use of a warning zone (ATU) for all CoNS, more than one 
susceptibility test, or a specialized molecular methodology may well be indicated. In conclusion, when adopting the BrCAST/EUCAST guidelines and comparing data during and after implementation, clinicians and institutions should be aware of the implications of the change.

\section{Compliance with ethical standards}

Conflict of interest The author d'Azevedo PA is member of the General Committee of BrCAST in 2019-2020.

\section{References}

1. May L, Klein EY, Rothman RE, Laxminarayan R (2014) Trends in antibiotic resistance in coagulase-negative staphylococci in the United States, 1999 to 2012. Antimicrob Agents Chemother 58: 1404-1409. https://doi.org/10.1128/AAC.01908-13

2. Becker K, Heilmann C, Peters G (2014) Coagulase-negative staphylococci. Clin Microbiol Rev 27:870-926. https://doi.org/10.1128/ CMR.00109-13

3. Pedroso SHSP, Sandes SHC, Filho RAT, Nunes AC, Serufo JC, Farias LM, Carvalho MAR, Bomfim MRQ, Santos SG (2018) Coagulase-negative staphylococci isolated from human bloodstream infections showed multidrug resistance profile. Microb Drug Resist 24:635-647. https://doi.org/10.1089/mdr.2017.0309

4. Clinical and Laboratory Standards Institute (CLSI) (2019) Performance standards for antimicrobial susceptibility testing. 29th informational supplement, MS100-S29. CLSI, Pennsylvania, USA

5. Clinical and Laboratory Standards Institute (CLSI/NCCLS) (2005) Performance standards for antimicrobial susceptibility testing. 15th informational supplement, M100-S15 [Normas de Desempenho para Testes de Sensibilidade Antimicrobiana: 15o Suplemento Informativo, M100-S15]. http://bvsms.saude.gov.br/bvs/ publicacoes/metodo_ref_testes_diluicao_modulo4.pdf. Accessed 15 January 2019

6. Brazil, Ministry of Health, Health Surveillance Secretary (2018) Ordinance no 64 of December 11, 2018 [Portaria no 64, de 11 de dezembro de 2018]. http://pesquisa.in.gov.br/imprensa/jsp/ visualiza/index.jsp?data $=14 / 12 / 2018 \&$ jornal $=515 \&$ pagina $=59$. Accessed 15 January 2019

7. Brazilian Committee on Antimicrobial Susceptibility Testing (BrCAST) (2013) Term of cooperation and creation of the committee [Termo de ooperação de criação do comitê]. http://brcast.org.br/ documentos/. Accessed 25 February 2019

8. European Committee on Antimicrobial Susceptibility Testing (EUCAST) (2017) Organization of General Committee.. http:// www.eucast.org/organization/general committee. Accessed 25 February 2019

9. Meneghetti KL, do Canto Canabarro M, Otton LM, dos Santos Hain T, Geimba MP, Corção G (2018) Bacterial contamination of human skin allografts and antimicrobial resistance: a skin bank problem. BMC Microbiol 18:121. https://doi.org/10.1186/s12866018-1261-1

10. European Committee on Antimicrobial Susceptibility Testing (EUCAST) (2019) Antimicrobial susceptibility testing: EUCAST disk diffusion method. version 7.0. http://www.eucast.org/ast_of bacteria/disk diffusion methodology/. Accessed 25 February $201 \overline{9}$

11. Clinical and Laboratory Standards Institute (CLSI) (2018) Performance standards for antimicrobial susceptibility testing. 28th informational supplement, MS100-S28. CLSI, Pennsylvania, USA
12. Brazilian Committee on Antimicrobial Susceptibility Testing (BrCAST) (2019) Breakpoint tables for interpretation of MICs and zone diameters [Tabelas de pontos de corte para interpretação de CIMs e diâmetros de halos]. Version 9.0. http://brcast.org.br/ documentos/. Accessed 25 February 2019

13. Lawung R, Chuong LV, Cherdtrakulkiat R, Srisarin A, Prachayasittikul V (2014) Revelation of staphylococcal cassette chromosome mec types in methicillin-resistant Staphylococcus aureus isolates from Thailand and Vietnam. J Microbiol Methods 107:8-12. https://doi.org/10.1016/j.mimet.2014.08.024

14. Argemi X, Riegel P, Lavigne T, Lefebvre N, Grandpré N, Hansmann Y, Jaulhac B, Prévost G, Schramm F (2015) Implementation of matrix-assisted laser desorption ionizationtime of flight mass spectrometry in routine clinical laboratories improves identification of coagulase-negative staphylococci and reveals the pathogenic role of Staphylococcus lugdunensis. J Clin Microbiol 53:2030-2036. https://doi.org/10.1128/JCM.00177-15

15. Hitzenbichler F, Simon M, Salzberger B, Hanses F (2017) Clinical significance of coagulase-negative staphylococci other than S. epidermidis blood stream isolates at a tertiary care hospital. Infection 45:179-186. https://doi.org/10.1007/s15010-016-0945-4

16. Widerström M, Wiström J, Sjöstedt A, Monsen T (2012) Coagulase-negative staphylococci: update on the molecular epidemiology and clinical presentation, with a focus on Staphylococcus epidermidis and Staphylococcus saprophyticus. Eur J Clin Microbiol Infect Dis 31:7-20. https://doi.org/10.1007/s10096011-1270-6

17. Lerbech AM, Opintan JA, Bekoe SO, Ahiabu M-A, Tersbøl BP, Hansen M, Brightson KTC, Ametepeh S, Frimodt-Møller N, Styrishave B (2014) Antibiotic exposure in a low-income country: screening urine samples for presence of antibiotics and antibiotic resistance in coagulase negative staphylococcal contaminants. PLoS One 9:e113055. https://doi.org/10.1371/journal.pone. 0113055

18. Misawa Y, Yoshida A, Okugawa S, Moriya K (2015) First reported case of Staphylococcus condimenti infection associated with catheter-related bacteraemia. New Microbes New Infect 3:18-20. https://doi.org/10.1016/j.nmni.2014.10.002

19. Khosravi AD, Roointan M, Abbasi Montazeri E, Aslani S, Hashemzadeh M, Taheri Soodejani M (2018) Application of tuf gene sequence analysis for the identification of species of coagulase-negative staphylococci in clinical samples and evaluation of their antimicrobial resistance pattern. Infect Drug Resist 11: 1275-1282. https://doi.org/10.2147/IDR.S172144

20. Czekaj T, Ciszewski M, Szewczyk EM (2015) Staphylococcus haemolyticus - an emerging threat in the twilight of the antibiotics age. Microbiol Read Engl 161:2061-2068. https://doi.org/10.1099/ mic. 0.000178

21. Cusack TP, Ashley EA, Ling CL, Rattanavong S, Roberts T, Turner P, Wangrangsimakul T, Dance DAB (2019) Impact of CLSI and EUCAST breakpoint discrepancies on reporting of antimicrobial susceptibility and AMR surveillance. Clin Microbiol Infect 25: 910-911. https://doi.org/10.1016/j.cmi.2019.03.007

22. Sánchez-Bautista A, Coy J, García-Shimizu P, Rodríguez JC (2018) Cambio de CLSI a EUCAST en la interpretación de la sensibilidad a antimicrobianos: ¿cómo influye en nuestro medio? Enfermedades Infecc Microbiol Clínica 36:229-232. https://doi.org/10.1016/j. eimc.2017.03.003

23. Marchese A, Esposito S, Barbieri R, Bassetti M, Debbia E (2012) Does the adoption of EUCAST susceptibility breakpoints affect the selection of antimicrobials to treat acute community-acquired respiratory tract infections? BMC Infect Dis 12:181. https://doi.org/10. 1186/1471-2334-12-181

24. Kassim A, Omuse G, Premji Z, Revathi G (2016) Comparison of clinical laboratory standards institute and European committee on antimicrobial susceptibility testing guidelines for the interpretation 
of antibiotic susceptibility at a university teaching hospital in Nairobi, Kenya: a cross-sectional study. Ann Clin Microbiol Antimicrob 15:21. https://doi.org/10.1186/s12941-016-0135-3

25. Farrell DJ, Mendes RE, Bensaci M (2019) In vitro activity of tedizolid against clinical isolates of Staphylococcus lugdunensis and Staphylococcus haemolyticus from Europe and the United States. Diagn Microbiol Infect Dis 93:85-88. https://doi.org/10. 1016/j.diagmicrobio.2018.08.006

26. Sader HS, Castanheira M, Farrell DJ, Flamm RK, Mendes RE, Jones RN (2016) Tigecycline antimicrobial activity tested against clinical bacteria from Latin American medical centres: results from SENTRY Antimicrobial Surveillance Program (2011-2014). Int J Antimicrob Agents 48:144-150. https://doi.org/10.1016/j. ijantimicag.2016.04.021

27. Pinheiro L, Mello PL, Abraão LM, Corrente JE, Lourdes RS, Cunha M d (2018) Evaluation of reference values for phenotypic tests to detect oxacillin resistance in coagulase-negative staphylococci. Future Microbiol 13:565-575. https://doi.org/10.2217/fmb2017-0221

28. Ferreira AM, Bonesso MF, Mondelli AL, Camargo CH, Cunha M d LRS (2012) Oxacillin resistance and antimicrobial susceptibility profile of Staphylococcus saprophyticus and other staphylococci isolated from patients with urinary tract infection. Chemotherapy 58:482-491. https://doi.org/10.1159/000346529

29. Le Bouter A, Leclercq R, Cattoir V (2011) Molecular basis of resistance to macrolides, lincosamides and streptogramins in Staphylococcus saprophyticus clinical isolates. Int J Antimicrob Agents 37:118-123. https://doi.org/10.1016/j.ijantimicag.2010.10. 008

30. Khashei R, Malekzadegan Y, Sedigh Ebrahim-Saraie H, Razavi Z (2018) Phenotypic and genotypic characterization of macrolide, lincosamide and streptogramin B resistance among clinical isolates of staphylococci in southwest of Iran. BMC Res Notes 11:711. https://doi.org/10.1186/s13104-018-3817-4

31. Teeraputon S, Santanirand P, Wongchai T, Songjang W, Lapsomthob N, Jaikrasun D, Toonkaew S, Tophon P (2017) Prevalence of methicillin resistance and macrolide-lincosamidestreptogramin B resistance in Staphylococcus haemolyticus among clinical strains at a tertiary-care hospital in Thailand. New Microbes New Infect 19:28-33. https://doi.org/10.1016/j.nmni. 2017.05.007

Publisher's note Springer Nature remains neutral with regard to jurisdictional claims in published maps and institutional affiliations. 